\title{
Design and Interpretation of Noninferiority Trials
}

\author{
Scott K. Aberegg, MD, MPH and Andrew M. Hersh, $\mathrm{MD}^{2}$ \\ 'Division of Pulmonary and Critical Care Medicine, University of Utah School of Medicine, Salt Lake City, UT, USA; ${ }^{2}$ Brooke Army Medical Center, San \\ Antonio, TX, USA.
}

J Gen Intern Med 33(8): 1217

DOI: $10.1007 / \mathrm{s} 11606-018-4505-8$

(c) Society of General Internal Medicine 2018

uthors' reply: We are grateful for the correspondents'
interest in our descriptive study of noninferiority trials. Turgeon et al. have retrospectively extended the part of our analysis comparing ITT (intention-to-treat) and PP (perprotocol) methodologies to 2004 . While we found that $64 \%$ of trials in our cohort from 2011 to 2016 reported both ITT and PP analyses, they found that only $45 \%$ of trials in the same journals between 2004 and 2014 reported both analyses. These results are consistent with those found in other reports, with modest increases over time in the reporting of both analyses. $^{2}{ }^{-4}$ Regarding discordant results between analyses, both of our results reinforce the observation that the theoretical claim that PP analyses are more conservative than ITT analyses is empirically ungrounded. Ideally, both PP and ITT methodologies should be carefully described and reported with an a priori plan for handling discordant results when they occur.

Vach et al. highlight our finding that a fundamental ethical tenet of noninferiority trials, namely that the NT (new treatment) have some secondary benefit, is frequently unmet. We regret that we neglected to reference their systematic review of this specific issue, which showed similar results to ours. ${ }^{5}$ In their study, $51 \%$ of trials explicitly reported the purported secondary advantages of the NT, and an additional $25 \%$ vaguely or indirectly mentioned secondary advantages. The corresponding percentages in our trial were 70 and $20 \%$. In their article, Vach et al. propose the integration of measures of benefits and harms of NT versus active control in a manner consistent with expected utility theory. This approach, which can be adapted at the level of the patient or the population, holds promise for shared decision-making using the results of randomized trials of all types. Application of these methods will be facilitated by reliable estimates of effects from well designed and executed trials, and from a focus on actual confidence intervals of beneficial and harmful effects, rather than arbitrary and potentially misleading conclusions such as "noninferior" or "inconclusive."

Corresponding Author: Scott K. Aberegg, MD, MPH; Division of Pulmonary and Critical Care Medicine University of Utah School of Medicine, Salt Lake City, UT, USA (e-mail: scottaberegg@gmail.com).

\section{Compliance with Ethical Standards:}

Conflict of Interest: The authors declare that they do not have a conflict of interest.

\section{REFERENCES}

1. Aberegg SK, Hersh AM, Samore MH. Empirical Consequences of Current Recommendations for the Design and Interpretation of Noninferiority Trials. Journal of general internal medicine 2018;33(1):88-96. https://doi. org/10.1007/s11606-017-4161-4

2. Wangge G, Klungel OH, Roes KC, et al. Room for improvement in conducting and reporting non-inferiority randomized controlled trials on drugs: a systematic review. PLoS One 2010;5(10):e13550. https://doi.org/ 10.1371/journal.pone.0013550 [published Online First: 2010/11/05]

3. Rehal S, Morris TP, Fielding $\mathbf{K}$, et al. Non-inferiority trials: are they inferior? A systematic review of reporting in major medical journals. BMJ Open 2016;6(10) https://doi.org/10.1136/bmjopen-2016-012594

4. Le Henanff A, Giraudeau B, Baron G, et al. Quality of reporting of noninferiority and equivalence randomized trials. JAMA 2006;295(10):1147-51. https://doi.org/10.1001/jama.295.10.1147 [published Online First: 2006/03/09]

5. Gladstone BP, Vach, W. Analyzing noninferiority trials: it is time for advantage deficit assessment-an observational study of published noninferiority trials. Open Access J Clin Trials 2015;2015(7):11-21. https:// doi.org/10.2147/OAJCT.S74821 